\title{
Oral anticancer therapy: a comprehensive assessment of patient perceptions and challenges
}

\author{
Paige May, PharmD, BCOP, ${ }^{a}$ and Bradley Figgins, $\mathrm{AA}^{\mathrm{b}^{*}}$
}

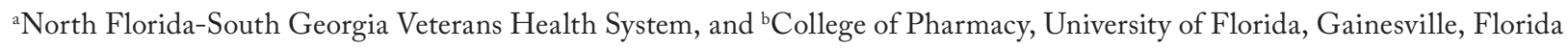

Background Oral anticancer agents are more convenient to use and better tolerated than traditional intravenous therapy but come with significant concerns about patient noncompliance, adverse effects, and high cost. Identifying areas for improvement in the medication use process may help ensure optimal use of these agents.

Objectives To characterize patient experience with oral anticancer treatment, highlight the areas for improvement in the medication use process, and assess the utility of a pharmacist-led educational program.

Methods 30 patients who were receiving oral anticancer therapy were administered a brief survey during their visits to an ambulatory Department of Veterans' Affairs oncology clinic where pharmacists are heavily involved in providing initial and follow-up medication use education. Veterans aged 18 years or older were considered for inclusion into the study if they were currently being treated with an oral anticancer medication from a specified list for at least 1 month. Topics addressed included drug information sources, regimen compliance, management of side effects, and cost. The results were results were analyzed using univariate descriptive statistics.

Results Most of the patients were satisfied with their oral treatment, reporting ease of use with minimal side effect occurrence. Oncologists and pharmacists were equally named as sources of drug information.

Limitations Sample size was small and patients were overwhelmingly male. Response bias may be partially responsible for the observed results for regimen management, side effect occurrence, missed doses, and overall treatment satisfaction.

Conclusion Oral anticancer therapy represents a significant therapeutic advance for many types of cancer. Pharmacists can serve as vital informational resources to these patients. Further studies examining the role of pharmacist-led educational programs in terms of overall patient outcomes are warranted.

A $\mathrm{n}$ important development in the new cancer care model is the recognition of cancer as a chronic ailment, rather than an acute, imminently life-threatening process. Historically, cancer treatment entailed rigorous intravenous therapy with poorly tolerated cytotoxic drugs in addition to radiation therapy and surgical interventions. Even with pursuit of these intensive, multimodal treatment strategies, survival statistics were abysmal and patient quality of life was severely affected by iatrogenic toxicities. Intravenous therapy is still a critical component of cancer treatment in today's practice, but a shift toward oral treatment for some cancers is becoming increasingly apparent. Although some traditional chemotherapy agents have been available in oral dosage forms for many years (eg, chlorambucil, 6-mercaptopurine, methotrexate), the modern era of oral chemotherapy was ushered in 1998 when the US Food and Drug Administration approved capecitabine. Advances in the pharmaceutical devel- opment process and a better understanding of the molecular aspects of cancer pathophysiology have spurred the discovery and approval of numerous target-specific, orally bioavailable small molecules for treatment of multiple solid and hematological malignancies. From 2011-2014, 25 new oral agents were granted FDA approval; in this same timeframe, just 18 new intravenous agents were approved. It is an ongoing trend, with multiple new oral agents receiving approval through 2015. It has been estimated that more than $25 \%$ of all hematology-oncology drugs currently in development are orally available small molecules. ${ }^{1}$ These drugs, in addition to significant improvements in supportive care measures, have greatly improved the quality and quantity of life for patients suffering from many different types of cancer. A revolutionary example resides in the use of oral BCR-ABL kinase inhibitors such as imatinib in Philadelphia chromosome-positive chronic myelogenous leukemia, once a leading indication for alloge-

Accepted for publication December 1, 2015. *PharmD candidate, 2016. Correspondence: Paige May, PharmD, BCOP; Paige.May@va.gov. Disclosures: The authors report no disclosures or conflicts of interest. JCSO 2016;14:112-116. (C2016 Frontline Medical Communications. doi: 10.12788/jcso.0226. 
neic stem-cell transplantation. However, these oral drugs do not come without significant risk and concern.

As the concept of patient-centered health care becomes increasingly prominent in today's practice of oncology, it is important to recognize and address some of the potential concerns with oral anticancer therapy in order to resolve problems with treatment and ultimately improve the quality of care the patient receives. Development of effective, multidisciplinary patient education strategies will be of paramount importance, as use of these medications is expected to increase with the aging baby boomer population and improving cancer survival prognoses.

Many of the tasks that are necessary to assess and improve patient experience with oral chemotherapy are the responsibility of the pharmacist working in collaboration with other members of the health care team. Given their extensive drug knowledge and unique skill set, pharmacists can play a critical role in providing education and ensuring regimen adherence. As physicians' schedules become increasingly strained, face-to-face communication time with patients can become very limited, and this presents a void that pharmacists may be able to fill.

The quality of the patient-provider relationship and communication along with the amount of patient education provided by the health care staff and the level of patient understanding can all influence patient compliance, safety, and overall experience with treatment. ${ }^{2}$ The Malcom Randall Veterans Affairs Medical Center in Gainesville, Florida, has an innovative model for pharmacist involvement in the care of oral chemotherapy patients. Oncology pharmacy specialists and pharmacy student interns are heavily engaged in counseling patients throughout their oral treatment during face-to-face interviews and periodic phone calls. Initial consultation interviews allow the pharmacist and/or student to review indications, dosing, potential side effects, and various other aspects of the new medication directly with the patient. Follow-up adherence calls are made to assess understanding and uncover any problems or side effects the patient may be experiencing between appointments.

The objective of this study was to investigate and describe patient and/or caregiver perceptions of oral anticancer treatment to uncover any obstacles or potential areas for improvement in several aspects of the medication use process. These include patient education, regimen adherence, use of drug information sources, and side-effect management. A survey-based investigation was conducted over 3 months in the outpatient oncology clinic within the Malcom Randall Veterans Affairs Medical Center to evaluate the individual patient experiences.

\section{Methods}

The study protocol and questionnaire were developed over several months in the summer of 2014. Approval from the University of Florida institutional review board (IRB) and the North Florida-South Georgia Veterans Affairs Research and Development Department was obtained in December of that year.

Veterans aged 18 years or older who presented to the medical center for an outpatient oncology visit between December 1, 2014-February 28, 2015 were considered for inclusion into the study if they were currently being treated with any of the following oral anticancer medications for at least 1 month: capecitabine, sunitinib, sorafenib, erlotinib, afatinib, pazopanib, vemurafenib, abiraterone, lenalidomide, pomalidomide, 6-mercaptopurine, enzalutamide, everolimus, temozolamide, ibrutinib, imatinib, dasatinib, or nilotinib. Patients were excluded only if they were unable to provide informed consent or if they had been receiving oral treatment for less than a month. Lists of potential participants were generated from weekly outpatient oral chemotherapy follow-up appointment schedules. The data collection period was set to finish once the enrollment goal of 50 patients was met or a span of 3 months passed, which ever occurred first.

After patients had been deemed eligible, they were approached by study personnel and invited to participate in the study. If the patients agreed, they were escorted to a private enclosed room where they were provided with more in-depth information about the study and their rights as a potential participant. Patients were notified explicitly that their responses would remain anonymous and have no effect on their current care. They were given the opportunity to ask questions about the study, after which they were asked to review and sign a jointly approved IRB-VA consent form. After informed consent had been obtained, patients were assigned a unique participant number and administered a 20-item questionnaire (Appendix 1) covering various aspects of their treatment. Questions varied in structure and included free-response, multiple-choice, and scale-type items. After completion, the patients were provided with a copy of their signed consent form and were free to leave. Additional demographic data (eg, age, gender, cancer diagnosis, medical record number, duration of oral treatment) was collected using the VA computerized patient record system (CPRS) and recorded on their answer sheets.

The study encounter was documented in the patient's medical record within the CPRS. All study-related documents were retained in a locked cabinet file to ensure their security and patient confidentiality. A list of participant names with assigned participant numbers was kept in a password-protected folder on the internal shared T-drive and was accessible only to study personnel. Responses from each patient interview were entered into an Excel spreadsheet for further analysis using descriptive statistics.

\section{Results}

Over the 3-month study period, 30 veterans agreed to participate and were successfully interviewed. Almost 

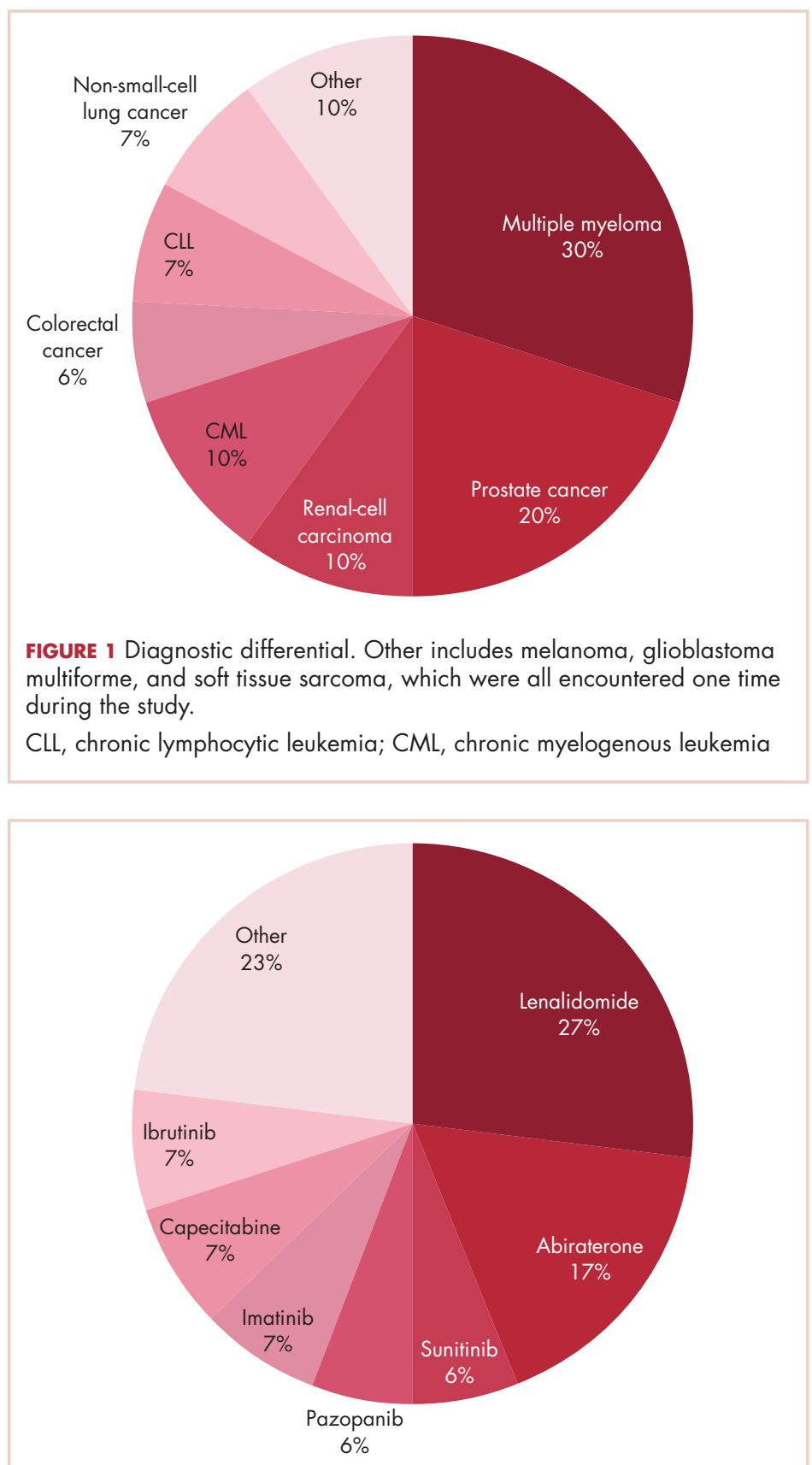

FIGURE 2 Drug differential. "Other" includes vemurafenib, pomalidomide, afatinib, erlotinib, enzalutamide, temozolamide, and dastinib, which were all encountered once during the study.

all of the patients were men (96.7\%), and the mean age was 68 years (range, 21-88 years). The average duration of treatment with an oral anticancer therapy was 6.2 months (range, 1-27 months). Diagnostic and drug differentials are shown in Figure 1 and Figure 2, respectively. The most common cancer diagnosis-drug combination was multiple myeloma and lenalidomide, followed closely by prostate cancer and abiraterone. An

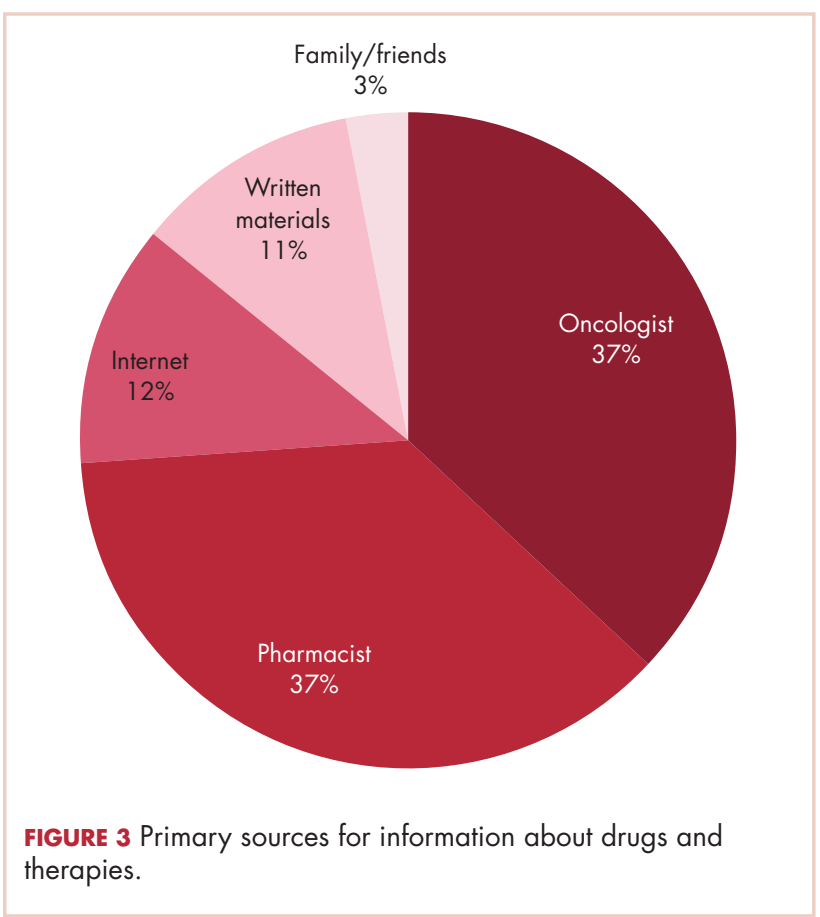

equal amount $(n=15)$ of hematological and solid tumor patients were included. The results of all 30 questionnaires were tabulated (Table 1 ).

When given a choice, patients chose oral therapy over intravenous therapy, most commonly because of the convenience. Of note, it was not always documented in the medical record whether patients were given a choice at all, and if so, what information was provided to the patient to help in making that decision. Survey responses on preference of intravenous or oral therapy relied heavily on patient understanding. Most of the patients (83.3\%) managed their treatment on their own, without the assistance of a family member or caregiver. Ten percent admitted to occasionally missing doses of their chemotherapy. Oncologists were most often named as patients' primary source of drug information, followed by pharmacists and online or printed media (Figure 3). Patients were not particularly concerned with side effects prior to starting their oral chemotherapy, though two-thirds of all participants reported experience with at least 1 significant side effect once treatment was initiated. The most common management strategy was the use of a prescription medication and consultation with an oncologist; however, more often than not, the side effect was ignored and left untreated. Most of the patients were aware of the oral chemotherapy's name, directions for its use, and the indication. A small minority (16\%) was able to accurately state the price of a 1-month supply.

\section{Discussion}

Some advantages of oral treatment include ease of admin- 
istration, less time away from family and work, and an increased sense of independence. Although these drugs offer additional treatment options and the potential for improved convenience, they also pose several challenges for patients and their health care providers alike. Common patient concerns include the difficulty of managing complex treatment regimens at home, the identification and management of adverse side effects, problems with obtaining and safely storing the drug, and the difficulty of procuring user-friendly sources of drug information. These issues are worsened by the fact that the responsibility of drug administration has been taken out of the hands of highly trained, skilled health care professionals and given over to the often inexperienced patient or caregiver. The safeguards and close monitoring that are normally used with intravenous therapy are no longer in place. In the home setting, medical attention may not be readily available, as in a hospital or infusion center. Although patients may perceive oral agents as being less toxic than intravenous treatment, severe adverse effects may still occur and must be promptly addressed. Gastrointestinal upset (eg, nausea, vomiting), constipation, diarrhea, dermatological reactions (eg, acneiform rash, hand-foot syndrome), mucositis, and peripheral neuropathy are among the most common adverse effects with oral treatment, though incidence rates are agent specific. Education regarding proper use of supportive care medications (eg, antiemetics, antidiarrheals) and indications for emergency medical attention are essential to maximize patient safety.

Adherence to the prescribed regimen may also be problematic, especially given the fact that many oral chemotherapy drugs are dosed with multiple capsules or tablets multiple times each day. Further complicating this are dietary stipulations (eg, take with or without food), potential drug interactions with concomitantly used medications (eg, proton pump inhibitors, histamine- 2 receptor antagonists, antibiotics), and special storage or handling precautions (eg, use of gloves, no splitting, crushing, or opening). Unlike antihypertensives or cholesterol-lowering agents, with which occasionally missing a dose may not be so consequential, noncompliance to a prescribed regimen of oral chemotherapy may have devastating effects on patient prognosis. ${ }^{3}$

Cost can also be a significant concern because most oral anticancer agents currently on the market are not generically available and can cost thousands of dollars each month. A study by Shih and colleagues showed that from 2001-2011, oral anticancer medication insurance payment cost per patient per month increased from $\$ 3,381$ to $\$ 7,370$; in this same time frame, the cost of IV targeted therapies actually decreased from $\$ 7,190$ to $\$ 7,001 .{ }^{4} \mathrm{With}$ these medications, pharmaceutical companies commonly sponsor support programs for patients requiring significant financial assistance. Although these programs may reach a substantial number of needy patients, they do not
TABLE Questionnaire results ( $N=30$ )

\begin{tabular}{|c|c|}
\hline Question topic & Response \\
\hline \multicolumn{2}{|l|}{ Choice of treatment } \\
\hline $\begin{array}{l}\text { No. of patients voluntarily choosing } \\
\text { oral over IV treatment }\end{array}$ & $9(30 \%)$ \\
\hline $\begin{array}{l}\text { Most common reason for electing } \\
\text { oral treatment }\end{array}$ & $\begin{array}{l}\text { Convenience }(n=5) \\
\text { Oncologist } \\
\text { recommended }(n=5)\end{array}$ \\
\hline \multicolumn{2}{|l|}{ Regimen management } \\
\hline $\begin{array}{l}\text { Average difficulty rating (scale of 1-10; } \\
1=\text { Effortless, } 10=\text { impossible) }\end{array}$ & 1.57 (range, 1-6) \\
\hline $\begin{array}{l}\text { Average confidence level } \\
\text { (scale of } 1-10 ; 1=\text { Not at all confident, } \\
10=\text { Extremely confident) }\end{array}$ & 9.33 (range, 7-10) \\
\hline $\begin{array}{l}\text { No. of patients managing their } \\
\text { own treatment }\end{array}$ & $26(83.3 \%)$ \\
\hline No. of patients admitting to missed doses & $3(10 \%)$ \\
\hline \multicolumn{2}{|l|}{ Side effects } \\
\hline $\begin{array}{l}\text { No. of patients reporting } 1 \text { or more } \\
\text { side effect(s) }\end{array}$ & $20(66.7 \%)$ \\
\hline $\begin{array}{l}\text { Initial level of side-effect fear } \\
\text { (scale of } 1-10 ; 1=\text { Not at all concerned, } \\
10=\text { Extremely fearful) }\end{array}$ & 3.1 (range, 1-8) \\
\hline \multicolumn{2}{|l|}{ Most common side effects, n (\%) } \\
\hline Nausea/vomiting & $7(23.3)$ \\
\hline Diarrhea & $5(16.7)$ \\
\hline Rash & $3(10.0)$ \\
\hline Mucositis & $2(6.7)$ \\
\hline Dizziness & $2(6.7)$ \\
\hline Hand-foot syndrome & $1(3.3)$ \\
\hline Peripheral neuropathy & $1(3.3)$ \\
\hline Headache & $1(3.3)$ \\
\hline Peripheral edema & $1(3.3)$ \\
\hline \multicolumn{2}{|l|}{ Management of side effect(s), $n$} \\
\hline Self-treatment with prescription medication & 5 \\
\hline Called oncologist & 4 \\
\hline Self-treatment with OTC medication & 1 \\
\hline Called pharmacist & 1 \\
\hline Did nothing & 10 \\
\hline \multicolumn{2}{|l|}{ Cost } \\
\hline $\begin{array}{l}\text { No. of patients accurately stating monthly } \\
\text { cost of treatment }\end{array}$ & $5(16.7 \%)$ \\
\hline
\end{tabular}

represent a complete solution and can be quite difficult for patients to navigate. Methods to improve adherence and optimize use of these medications may also represent a source of cost-savings for the increasingly strained US health care system.

Multiple myeloma was the most frequently encountered diagnosis, and lenalidomide the most commonly encountered medication. This could be attributed to the presence of several multiple myeloma clinical special- 
ists within the Gainesville area, in addition to the fact that the study population was overwhelmingly elderly and male, both defined multiple myeloma risk factors. ${ }^{5} \mathrm{~A}$ 2014 epidemiological review analyzing several pieces of currently available evidence concluded that past exposure to Agent Orange (2,3,7,8-tetrachlorodibenzo-p-dioxin), a well-documented carcinogen, may be associated with an increased lifetime risk of developing multiple myeloma. ${ }^{6}$ Given the veteran population, this could also be a potential explanation.

Limitations of the study include the homogeneity of the sample population (mostly white elderly males), small sample size, and probable response bias. Given the inherent characteristics of the veteran patient population, it is difficult to design a VA study that is easily generalizable to the overall patient population. Women, minorities, and young people are all underrepresented in our study, as expected. Only 1 woman was enrolled into the study. Given the near complete lack of female participants, we were not able to examine the use of oral therapy for breast cancer, a disease for which 3 new oral agents have been approved in the past 8 years (lapatinib, 2007; everolimus, 2012; and palbociclib, 2015). Future studies specifically targeting this patient population are warranted.

Although measures to ensure patient comfort and honesty during the interview process were in place, it is probable that responses to some questions may have been a result of patients' inherent desire to please their provid-

\section{References}

1. Weingart SN, Brown E, Bach PB, Eng K, Johnson SA, Kuzel TM et al. NCCN Task Force Report: oral chemotherapy. J Natl Compre Cancer Netw. 2008;6:S1-14.

2. Bedell CH. A changing paradigm for cancer treatment: the advent of new oral chemotherapy agents. Clin J Oncol Nurs. 2003;7(suppl 6):5-9.

3. Partridge AH, Avorn J, Wang PS, Winer EP. Adherence to therapy with oral antineoplastic agents. J Natl Cancer Inst, 2002;94:652-61.

4. Shih YC, Smieliauskas F, Geynisman DM, Kelly RJ, Smith

TJ. Trends in the cost and use of targeted cancer therapies for ers. This may have partially affected the results for overall regimen management confidence level, initial side-effect fear level, and missed doses. For some patients, sideeffect assessment may have been clouded by concurrent intravenous treatment. Nevertheless, it is of concern that nearly half of the participants stated that they did nothing when an adverse side effect was experienced. That may have been partially attributable to the misconception that oral treatment is devoid of serious toxicities, which was verified by the low initial fear rating (3.1/10) seen in our investigation. This suggests a potential area for clarification when patients are educated about the drugs before initiation of oral therapy.

\section{Conclusion}

In summary, results indicate that patients are generally able to manage their oral anticancer therapy well. As outpatient treatment with oral therapy becomes a more prominent treatment modality in the area of hematology and oncology, it will be increasingly important to ensure that patients have a complete and accurate understanding of their medications to ensure safety and optimal outcomes. Given that patients named pharmacists as a major source of drug information, it would be useful to conduct additional studies on the role of the pharmacist in improving patient outcomes. Additional studies directly comparing patients who received pharmacist-led education to those who did not receive such education would be of use in further characterizing potential benefits.

the privately insured nonelderly: 2001 to 2011 . J Clin Oncol. 2014;58:2320-2329.

5. Ferlay J, Soerjomataram I, Ervik M, et al. GLOBOCAN 2012: Estimated cancer incidence, mortality and prevalence worldwide in 2012. International Agency for Research on Cancer, 2013. http:// globocan.iarc.fr. Accessed February 18, 2016.

6. Chang ET, Boffetta P, Adami HO, Mandel JS. A critical review of the epidemiology of Agent Orange or 2,3,7,8-tetrachlorodibenzo- $p$-dioxin and lymphoid malignancies. Ann Epidemiol. 2015;25:275-292. 